\title{
O salto qualitativo em Theodosius Dobzhansky: unindo as tradições naturalista e experimentalista
}

A quality leap in Theodosius Dobzhansky: uniting naturalistic and experimental traditions
ARAÚJO, A. M. de: 'O salto qualitativo em Theodosius Dobzhansky: unindo as tradições naturalista e experimentalista'.

História, Ciências, Saúde-Manguinhos, vol. VIII(3): 713-26, set.-dez. 2001.

Em alta durante quase todo o século XIX, o prestígio dos naturalistas caiu nas últimas décadas do mesmo século, na medida em que o enfoque dos estudos biológicos transitava do histórico para o funcional. A redescoberta dos trabalhos de Mendel, em 1900, e a emergência da genética aceleraram este processo, onde a atividade do experimentalista substituía a anterior. Ao mesmo tempo, o darwinismo também declinava e os primeiros anos do século XX caracterizam o chamado "eclipse do darwinismo". Theodosius Dobzhansky, russo radicado nos Estados Unidos a partir de 1927, é o pesquisador que irá reunir as duas tradições, a naturalista e a experimentalista. No desempenho de suas atividades, ele realiza um verdadeiro salto qualitativo nos estudos de evolução em populações naturais, que pela primeira vez podem ser efetuados através do método experimental, auxiliados por um rigoroso planejamento teórico. Este artigo narra algumas etapas desta história.

PALAVRAS-CHAVE: evolucionismo, darwinismo, Theodosius Dobzhansky, naturalismo, experimentalismo, Mendel.

ARAÚJO, A. M. de: 'A quality leap in Theodosius Dobzhansky: uniting naturalistic and experimental traditions'.

História, Ciências, Saúde-Manguinhos, vol. VIII(3): 713-26, Sept.-Dec. 2001.

The high prestige naturalists had during most of the $19^{\text {h }}$ century began to decrease in the last decades of that century, as the focus in biological studies switched from historical to functional. The rediscovering of Mendel's works in 1900 and the emerging of genetics made the process move faster and caused experimentalist activities to take over. Simultaneously, Darwinism was also losing ground and the first years of the $2 O^{h}$ century were characterized by the so-called 'eclipse of Darwinism'. Theodosius Dobzhansky, a Russian researcher who moved to the United States in 1927, joined the two different traditional approaches, the naturalistic and the experimental ones. Through his activities, be accomplishes a quality leap for the study of evolution in natural populations, which can for the first time be carried out through experimental methods guided by thorough theoretical planning. This article narrates some of the steps of this story.

KEYWORDS: Evolutionism, Darwinism, Theodosius Dobzhansky, Naturalism, Experimentalism, Mendel.
Departamento de Genética e Grupo Interdisciplinar em Filosofia e História das Ciências, Universidade Federal do Rio Grande do Sul (UFRS) Caixa Postal, 15053

91501-970, Porto Alegre — RS Brasil aldomel@portoweb.com.br 


\section{A crise no darwinismo e a ascensão dos experimentalistas}

uando Charles Darwin nasceu, no ano de 1809, a atividade de
naturalista já tinha longa tradição na Europa. Certamente ele deu-he prestígio com a publicação de suas inúmeras obras, de um modo especial com $A$ origem das espécies (1859), onde desenvolvia o longo argumento da "descendência com modificações" e propunha o mecanismo para estas modificações, a seleção natural. Curiosamente a reputação de Darwin como grande naturalista, quem sabe representando o apogeu desta tradição, facilitou a emergência, na biologia, da tradição experimentalista, em prejuízo da anterior. Como sustentou Coleman (1977), os últimos 25 anos do século XIX testemunharam uma mudança de interesses na biologia. À medida que o enfoque da função orgânica substituía o enfoque da explanação histórica, o ideal da experimentação ganhava força. Alguns objetivos tradicionais da fisiologia, tais como o controle experimental de fenômenos, sua natureza quantitativa, bem como a possibilidade de previsão, estenderam-se praticamente a todas as áreas da biologia. Este conjunto de objetivos permitiu que Thomas Hunt Morgan assim se expressasse em 1904 (Coleman, 1977, p. 162):

O reconhecimento de que somente por métodos experimentais podemos ter a esperança de colocar o estudo da zoologia no mesmo nível de ciências como a química e a física é uma concepção relativamente nova ... . Creio que geralmente se admite que na atualidade há maior necessidade de trabalhos experimentais do que de estudos descritivos e observacionais (sic).

Uma contribuição decisiva para esta ruptura com o enfoque histórico foi o nascimento da genética, em 1900 (a denominação foi sugerida por William Bateson, em 1905, em carta enviada a Adam Sedgwick, professor da Universidade de Cambridge, solicitando recursos para a nova área). O uso de determinados cruzamentos, escolhidos pelo pesquisador, e o emprego de controles permitiam prever quais tipos de descendência deveriam ocorrer. Morgan, citado anteriormente, tornar-se-ia um dos expoentes da nova área.

O evolucionismo, originariamente associado ao darwinismo, seria caracterizado, nas primeiras décadas do século XX, como uma teoria ameaçada de extinção, especialmente quanto ao mecanismo proposto por Darwin, a seleção natural. No lugar desta, outros processos foram propostos, tais como a preponderância das mutações ("mutacionismo"), a ocorrência de evolução orientada para determinada direção, como, por exemplo, uma tendência ao aumento do tamanho corporal. Estas teorias que sustentavam um desdobramento de fatores internos na evolução ficaram conhecidas com os nomes de "ortogênese", "aristogênese", "nomogênese", entre outros. Coletivamente este movimento de descrédito do darwinismo ficou conhecido como "o 
eclipse do darwinismo", expressão criada por Julian Huxley (1943) ao descrever o surgimento de uma nova tendência em estudos evolutivos, a qual ele denominou de "nova síntese" e que hoje é conhecida como "teoria sintética da evolução". Tão forte foi o declínio do darwinismo que induziu a publicação de livros com títulos contundentes, como $A t$ the deathbead of Darwinism (Dennert, 1904, citado em Bowler, 1989). O interessante é que, depois de décadas de expansão no século atual, o darwinismo, representado pela teoria sintética da evolução, esteja sendo novamente questionado neste final de milênio; por ora é suficiente lembrar a publicação, no Brasil, do livro A caixa-preta de Darwin (Behe, 1997), uma tentativa de propor a idéia do "planejamento inteligente" na evolução. Na mesma linha está o livro de Rosine Chandebois (1993).

Uma das conseqüências do avanço das ciências experimentais, em particular da genética, foi o estabelecimento de um intenso debate sobre a natureza das mudanças evolutivas. Como se sabe, Darwin propunha que estas seriam graduais e contínuas; o problema é que ele desconhecia a causa das variações, as "leis" da herança, o que o fez adotar hipóteses como a da herança dos caracteres adquiridos, incorporando-a em sua hipótese da "pangênese". A incipiente área do conhecimento, então chamada mendelismo e, logo, genética, vinha mostrando, ao contrário, que as mudanças nos organismos eram descontínuas, envolvendo diferenças qualitativas. $\mathrm{O}$ conflito entre os chamados biometristas e mendelistas representou, na realidade, o auge de uma discórdia surgida ainda no século XIX. O grande defensor do darwinismo, Thomas Henry Huxley, por exemplo, afirmara, em carta enviada a Darwin após a leitura de A origem das espécies que (Provine, 1971, p. 12): "Você se impôs uma dificuldade desnecessária ao adotar o Natura non facit saltum tão indiscriminadamente".

Huxley, Galton e Bateson, todos duvidavam da efetividade da seleção natural atuando sobre pequenas variações. Galton, por exemplo, acreditava que a seleção sobre a variação contínua rapidamente chegaria a um limite, em virtude da "lei da regressão", proposta por ele ao analisar a variabilidade de características como estatura, doenças, capacidade artística etc. em humanos (ocasionalmente ele utilizou dados de outros organismos). Esta lei da regressão estabelecia que em populações onde as características permanecem constantes durante as gerações (na verdade, a média) haveria, mesmo assim, uma certa amplitude de variação a cada geração. No entanto, sustentava Galton, esta amplitude seria a mesma, a cada geração. Dessa forma, os indivíduos mais extremos de uma geração não poderiam produzir descendentes mais extremos. Entretanto, uma vez que indivíduos médios poderiam produzir indivíduos extremos, estes, por sua vez, tenderiam a produzir uma prole menos extrema. Esta é a essência da regressão, do retorno à média da população. Como ele próprio referiu em Natural Inheritance (1889, citado em Provine, 1971, p. 21): "Se $\mathrm{P} \pm \mathrm{D}$ for a estatura dos pais, 
a estatura da prole será, na média, $\mathrm{P} \pm 1 / 3 \mathrm{D}$.” Neste caso, P é a média populacional e D, o desvio dos pais em relação a esta média (Figura 1).

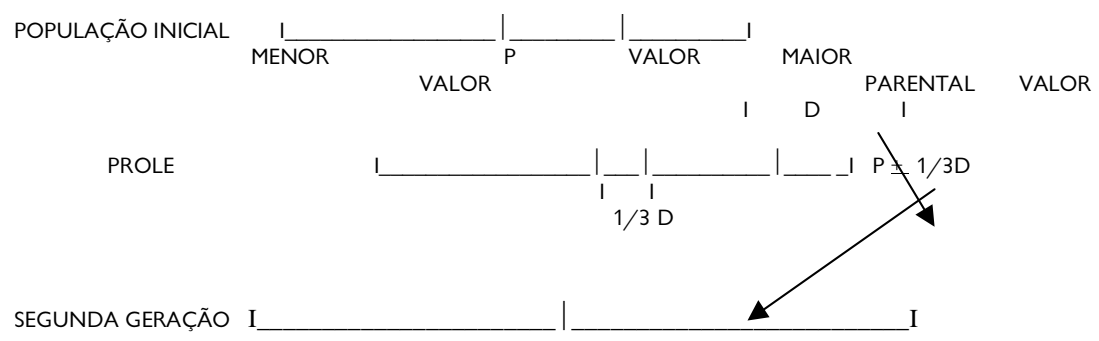

Figura 1 - Representação esquemática das conseqüências da “lei da regressão", enunciada por Francis Galton (1889). As setas indicam a produção eventual de prole cujo valor difere muito dos pais, mas que regride na geração seguinte.

Hugo De Vries, o proponente do mutacionismo como alternativa para o darwinismo, adicionou o suporte experimental que os três pesquisadores anteriores necessitavam; seus experimentos mostraram que a seleção era pouco efetiva ao operar sobre a variação contínua e que apenas mutações de grande efeito seriam importantes na evolução. O dinamarquês Wilhelm Johannsen foi outro pesquisador que utilizou a experimentação para comparar seleção com a lei da regressão de Galton. Ele fez uso das chamadas "linhas puras", isto é, proles produzidas por um único indivíduo autofecundado, utilizando uma variedade de feijão, o Phaseolus vulgaris: Johannsen acreditava que estas linhas puras representariam o caso mais simples e que, se fossem entendidas, uma teoria geral poderia ser proposta. Ele mostrou, ao longo de experimentos realizados em 1901 e 1902, que a lei da regressão não se aplicava às linhas puras, ainda que fosse válida para a população total, composta de várias linhas puras. Como se sabe, os experimentos de Johannsen forneceram a base para a teoria da herança poligênica; através desta teoria se estabeleceu que caracteres contínuos podem ser explicados pela herança de inúmeros fatores, os "poligenes". A outra contribuição de Johannsen foi a elaboração dos conceitos de "fenótipo" e "genótipo". Em uma linha pura os indivíduos poderiam ter fenótipos diferentes (resultado, em certa medida, de ambientes ligeiramente diferentes), mas os genótipos seriam os mesmos, daí a inatividade da seleção.

Outras evidências experimentais a favor dos resultados de Johannsen provinham dos Estados Unidos; Herbert Spencer Jennings, trabalhando com o protozoário Paramecium, e Raymond Pearl, com aves domésticas, nos anos de 1906 a 1908, estabeleceram o conceito de que a seleção não era efetiva sobre linhas puras. Seus trabalhos foram muito citados como apoio aos resultados de Johannsen, de tal forma que, por volta de 1910, poucos geneticistas sustentavam o contrário. Todavia, tanto os experimentos de Jennings quanto os de Pearl foram criticados por Karl Pearson, o grande teórico e defensor dos biometristas, principalmente 
no que se referia à estimativas quanto à possibilidade de serem herdadas as variáveis empregadas por ambos (Provine, 1971).

O conflito entre mendelistas e biometristas tornou-se mais brando ao longo da década de 1910, devido a uma série de outros experimentos, tanto em vegetais como em animais; dentre estes, merecem destaque, por sua relação com o que virá a seguir, os experimentos com a mosca Drosophila. O responsável pela introdução deste novo material de pesquisa experimental parece ter sido William Castle, da Universidade de Harvard, em 1901. Thomas H. Morgan, o nome geralmente associado à pesquisa com Drosophila melanogaster, passou a usá-la a partir de 1906 (uma excelente discussão e análise histórica da Drosophila como material de estudo foi feita por Kohler, 1994, em Lords of the Fly, uma inteligente paródia do romance do escritor inglês William Golding, Lord of the Flies. Morgan, um crítico do darwinismo no início de sua vida acadêmica e, depois, também da abordagem histórica e descritiva da biologia, descobriu, assim que começou a trabalhar com Drosophila, que pequenas variações eram passíveis de serem herdadas. Como ele próprio afirmou em artigo de 1909, esta constatação favorecia a concepção darwiniana de evolução: "Nós descobrimos ... que algumas 'pequenas variações' são herdadas. Vamos chamá-las de 'variações definidas' e, se este for o material com o qual a evolução trabalha, então a suposição de Darwin em relação à natureza da variação será, em parte, justificada" (Provine, 1971, p. 120).

Esta "conversão" de Morgan acentuou-se à medida que ele e seu grupo prosseguiam nas pesquisas sobre herança de características morfológicas em Drosophila. É preciso que se diga que Morgan, um zoólogo eclético até encontrar a Drosophila, também rejeitava, de início, o mendelismo e a teoria cromossômica da herança. A mudança teria começado a ocorrer em 1910, quando ele constituiu um grupo com três estudantes da Universidade de Columbia, mais tarde referidos como integrantes da fly room, internacionalmente conhecida. Os três estudantes foram Alfred Sturtevant, Calvin Bridges e Herman Muller, que viriam a ter grande reconhecimento como geneticistas, especialmente Sturtevant e Muller, este também ganhador do prêmio Nobel, como Morgan. Os antecedentes de Morgan e o processo de conversão, bem como uma hipótese para explicá-la foram muito bem explorados por Martins (1998).

O ano de 1918 é usualmente referido como representando o término da disputa entre biometristas e mendelistas. Alguns aspectos teóricos da incipiente genética de populações começavam a ser resolvidos; um deles, mais precocemente, foi o da conservação da variabilidade genética nas populações, independentemente do fato de ser esta devida a fatores ditos dominantes ou recessivos. Como demonstraram em 1908 o matemático inglês G. Hardy e o médico alemão W. Weinberg, em artigos independentes, dadas algumas condições, uma população manteria inalteradas a freqüência de determinados fatores genéticos (o que hoje chamamos freqüências alélicas e freqüências genotípicas). 
A contribuição de ambos ficou conhecida como o "equilibrio de HardyWeinberg”. Em 1915, o inglês R. C. Punnett publicou um livro sobre mimetismo em borboletas onde incluiu uma tabela especial, solicitada ao matemático H. T. J. Norton, a qual mostra que pequenas intensidades de seleção natural, atuando em poucas gerações, podem alterar em muito as freqüências dos fatores que induzem a formação de borboletas miméticas. Finalmente, Fisher (1918) publica famoso artigo, 'The correlation between relatives on the supposition of Mendelian inheritance', em que diz:

Numerosas investigações realizadas por Galton e Pearson mostraram que todo o ambiente mensurável tem muito menos efeito sobre medidas como a estatura. Mais ainda, os fatos coletados por Galton em relação a gêmeos idênticos mostram que, neste caso, onde a natureza essencial é a mesma, a variância é muito menor. A hipótese mais simples, aquela que vamos examinar aqui, é que tais características, como a estatura, são determinadas por um grande número de fatores mendelianos e que a variância entre filhos dos mesmos pais é devida à segregação daqueles fatores, cujos pais são heterozigotos.

Nada poderia ser mais explícito do que igualar mendelismo, que é resultado da ação de poucos fatores, com características biométricas, que resultam da ação de muitos fatores. Hoje em dia a herança mendeliana pode ser vista como um caso particular da herança poligênica; além do mais, do ponto de vista estatístico, sabemos hoje que uma distribuição binomial pode se converter em distribuição normal e viceversa, dependendo do valor do expoente, n, do binômio.

O resumo dos principais eventos relacionados à evolução biológica nos anos de 1900 a 1918 foi usado como moldura para salientar dois pontos importantes: o primeiro, que a teoria darwiniana, a qual na sua versão contemporânea é aceita pela quase totalidade dos biólogos, esteve por algum tempo ameaçada de extinção, particularmente quanto ao seu principal processo, a seleção natural. O segundo aspecto que foi levado em conta neste resumo foi tornar evidente o declínio da credibilidade do naturalista como estudioso da evolução, em contrapartida ao crescente prestígio do experimentalista. Poucos expressaram tão bem este contraste como o fisiologista de Harvard W. J. Crozier, o qual, na década de 1920, costumava afirmar a seus estudantes (Smocovitis, 1996, p. 118): "Evolução é um bom tema para suplementos de domingo nos jornais, mas não é ciência. Você não pode fazer experimentos com dois milhões de anos!".

Este é o cenário em que vivia, ainda que ignorando uma grande parte destas questões, um jovem biólogo russo chamado Theodosius Dobzhansky. 


\section{Dobzhansky, o naturalista}

Theodosius Dobzhansky nasceu em 25 de janeiro de 1900, na cidade de Nemirov, distante cerca de $200 \mathrm{~km}$ de Kiev. Seu pai, Gregory Dobzhansky, era professor de matemática na escola secundária; sua mãe, Sophie Dobzhansky, era dona de casa. Ambos eram ligados à Igreja Ortodoxa Russa; Sophie era, inclusive, filha e neta de reverendos desta Igreja. Nos primeiros dez anos de casados não tiveram filhos, o que os levou a visitar vários locais religiosos da Rússia, para rezar no sentido de terem um filho. Num destes locais, Chenigov, oraram para são Theodosius, prometendo-lhe dar o mesmo nome ao filho que viessem a ter (Land, 1973).

A educação primária de Dobzhansky foi feita por professores particulares em sua própria casa, como era hábito nas famílias de classe média da época. Aos nove anos de idade, quando ingressou em uma escola pública, já era um naturalista incipiente, sendo colecionador de borboletas (hábito que cultivaria por vários anos). Por volta de 1914-15, já decidido a se tornar biólogo e residindo em Kiev, leu, junto com seu grande amigo Vadim Alexandrovsky, A origem das espécies, de Darwin. O encontro com um entomólogo amador, Victor Luchnik, induziu-o a deixar de lado as borboletas e se dedicar aos besouros (coleópteros) da família Coccinellidae, visando tornar-se um especialista (Land, 1973). A entomologia era uma das disciplinas biológicas mais desenvolvidas na Rússia nos primeiros 25 anos do século XX; havia forte interação entre profissionais e amadores dos grandes centros e de pequenas localidades (Krementsov, 1994). A pesquisa com besouros levou-o a publicar o primeiro trabalho em 1918, sobre a descrição de uma nova espécie de Coccinella dos arredores de Kiev; a paixão pelos coleópteros continuaria ainda por muito tempo em sua vida, mesmo depois de se tornar um geneticista internacionalmente conhecido pelos seus trabalhos com Drosophila, pois publicou vários estudos sobre aqueles organismos, o último deles em 1941.

Dobzhansky graduou-se em biologia em 1921, pela Universidade de Kiev; no mesmo ano tornou-se professor assistente de zoologia no Instituto Politécnico de Kiev, e, em 1924, a convite do prof. Iuri Filipchenko, transferiu-se, como docente, para a Universidade de Leningrado; naquele ano ele publicaria o seu primeiro trabalho sobre Drosophila melanogaster. No verão de 1926 e no de 1927, Dobzhansky realizou pesquisas sobre animais domésticos no Casaquistão, como parte de suas atividades para a Academia de Ciências, onde obtivera um segundo emprego, visando aumentar seus rendimentos. Destas atividades ele publicou um extenso relatório, com mais de duzentas páginas, para a Academia de Ciências da União Soviética, em 1928. Um ano antes, ele e a esposa Natasha haviam visitado o Turquestão, durante as férias de verão. Foi então que ele descobriu uma espécie de besouro que ainda não conhecia e que hibernava em grupos de 
dezenas de milhares de indivíduos. A descoberta era inédita para a família Coccinellidae e Dobzhansky publicou mais tarde um trabalho sobre o assunto, em periódico alemão.

Iuri Filipchenko foi um personagem importantíssimo na vida acadêmica de Dobzhansky; ele não apenas o convidou para lecionar em Leningrado (então chamada de Petrogrado), mas, devido ao seu relacionamento com Thomas Hunt Morgan, o indicou para estagiar no laboratório deste pesquisador, visando um retorno à União Soviética para liderar um grupo de pesquisadores em trabalhos com a mosca Drosophila. No dia 1o de dezembro de 1927, Morgan escreveu uma pequena carta a Dobzhansky, onde dizia:

Prezado dr. Dobzhansky:

Estou muito contente de saber que podemos ter a esperança de tê-lo aqui em breve. Sei das dificuldades em obter um passaporte - a demora, como eu entendo, deve-se ao nosso próprio consulado. Estamos prontos a recebê-lo em qualquer época e aguardamos com interesse sua vinda. Sinceramente,

T. H. Morgan

No dia 27 do mesmo mês, Dobzhansky e sua esposa Natasha chegaram aos Estados Unidos.

\section{Rompendo a dicotomia naturalista/experimentalista}

Os primeiros anos de Dobzhansky nos Estados Unidos caracterizaramse por intensa atividade de laboratório, praticamente desvinculada do seu grande objetivo de vida, que foi o de entender a evolução biológica e, em particular, de como a humanidade se situava neste quadro. Dois comentários de Dobzhansky podem ser citados para ilustrar o seu interesse em evolução (entrevista concedida a Garland Allen, 1994):

O meu interesse na genética veio do meu interesse na evolução - o meu interesse na evolução, posso dizer, era filosófico, veio primeiro; o interesse na genética veio dele. Assim, eu não tinha dúvidas desde o começo de que isto era o que queria fazer.

Na Rússia, evolução era considerado um tema da mais alta importância, tanto filosoficamente como sociologicamente. Você pode ou não saber, mas para um bom número de naturalistas e pensadores russos no século XIX, Darwin não era apenas uma teoria científica, mas a base para toda uma filosofia.

Este primeiro período, de 1928 até meados de 1932, pode ser considerado como a "morganização" de Dobzhansky (Allen, 1994). De fato, é neste período que ele se familiariza e domina as técnicas de cultivo e de experimentos com Drosophila. O ambiente no laboratório 
de Morgan, a "economia moral" do grupo (Kohler, 1994), caracterizava-se por intensa troca de idéias e discussão de problemas; o modo de produção era coletivo, essencialmente experimental. Havia fortes críticas e mesmo desconsideração pela atividade discursiva e especulativa atribuída aos naturalistas. Dobzhansky publicou muito, individualmente e em co-autoria; seu primeiro trabalho nesta nova fase foi de 1928, tratando dos efeitos da temperatura sobre a sobrevivência de um tipo especial de fêmeas (chamadas "superfêmeas" devido a uma constituição cromossômica especial) de Drosophila melanogaster. No mesmo ano, ele publicou outro trabalho, em colaboração com Calvin Bridges. Até 1932, ele publicou 22 trabalhos, como único autor ou em co-autoria, onde a Drosophila melanogaster é o principal objeto de estudo em 19 deles. Naquele ano, 1932, dois eventos mudaram substancialmente a linha de pesquisa de Dobzhansky: o primeiro deles, relativo à mudança de material experimental, isto é, de Drosophila melanogaster para Drosophila pseudoobscura, a qual, a partir daí, tornar-se-ia seu material preferencial. O segundo acontecimento foi o encontro com Sewall Wright, um dos grandes teóricos da genética de populações e da evolução. Dobzhansky e Wright encontraramse por ocasião do Sexto Congresso Internacional de Genética, realizado em Ithaca, estado de Nova York. Neste congresso, Wright apresentou o célebre trabalho sobre os papéis da mutação, endocruzamento, exocruzamento e seleção natural, onde aparecem as figuras com os picos e vales adaptativos que Dobzhansky divulgaria em Genetics and the origin of species, de 1937 . Vale a pena fazer aqui uma curta digressão, para registrar que o único brasileiro presente ao congresso, Carlos Arnaldo Krug, do Instituto Agronômico de Campinas, publicou um artigo na Revista de Agricultura (Piracicaba), no mesmo ano, comentando vários aspectos deste congresso. Como ele diz na p. 452 do artigo:

A exposição de material vivo e em preparações também foi de grande sucesso, ilustrando variados estudos de genética e a sua aplicação no melhoramento. Sem dúvida, a de maior interesse foi a de Drosophila, cuja genética tem sido estudada mais de que qualquer outro animal ou vegetal. No grande Laboratorio de Genética achavamse, no dia marcado pelo programa para demonstração da Drosophila, quase todos os pesquisadores deste díptero, tais como: T. H. Morgan, C. B. Bridges, A. H. Sturtevant, M. Demerec, H. J. Muller, A. Weinstein, Curt Stern, Timoféeff-Ressowsky, Jack Schultz, C. Zeleny, Dobzhansky e outros, cada um demonstrando os seus 'stocks' de moscas e muitos, também excelente material citológico ilustrando a espermatogênese, oogênese, mitose somática, anomalias cromossômicas (translocações, duplicações, inversões etc.); para este fim foram montados no laboratório cerca de cinqüenta microscópios de precisão. 
Ainda no citado artigo (p. 449), Krug registra que estava inscrito no congresso o trabalho do prof. Salvador de Toledo Piza, de Piracicaba, sobre Conservation of a morphological individuality of the chromosomes at the resting nucleus: "Infelizmente este técnico patrício não pôde comparecer ao congresso, onde não somente teria apresentado em pessoa o seu trabalho, como tambem poderia tê-lo demonstrado com suas micropreparações."

A troca de material experimental ficou registrada na publicação de três artigos, em 1933, com Drosophila pseudoobscura, onde Dobzhansky é o único autor em dois deles. O tema é o da esterilidade de híbridos inter-raciais e do papel dos autossomos nos híbridos desta espécie. De grande interesse é, no entanto, o artigo que ele publica, no mesmo ano, sobre variação geográfica em besouros. A maior parte dos dados ainda é do seu período de naturalista na então União Soviética; são 130 páginas relatando a variabilidade intra e interespecífica, com o uso de uma linguagem típica dos naturalistas; pode-se especular sobre a repercussão que terão tido entre seus colegas de laboratório algumas conclusões que o trabalho apresenta, como, por exemplo:

Seria muito longo discutir aqui as causas possíveis da diferenciação de espécies em raças, distinguidas umas das outras pela freqüência relativa de diferentes biótipos. Três explanações podem ser mencionadas aqui. Primeiro, uma população originalmente homogênea estendendo a sua distribuição geográfica pode tornarse diferenciada de acordo com as condições ambientais prevalentes nas diferentes partes da área. Segundo, vários biótipos podem surgir por mutação; as mutações podem ser igualmente freqüentes em todas as partes de uma área específica, mas algumas delas tornam-se estabelecidas e outras rejeitadas pela seleção natural. Diferentes mutações podem ser favorecidas em diferentes regiões. Terceiro, os fenômenos observados podem ser parcialmente explicados supondo hibridação entre duas ou mais raças preexistentes, cada uma das quais, antes da hibridação, sendo caracterizada por um padrão ou padrões de cores definidas. De qualquer modo, não há necessidade de se supor que o padrão de coloração seja, ele próprio, o caráter sobre o qual atua a seleção natural (p. 124).

Ainda que este trabalho com besouros tenha sido executado dentro de uma concepção de biologia evolutiva, ele apresentava duas dificuldades maiores: a primeira, em relação à ausência de discussão sobre a dinâmica dos processos de diferenciação geográfica; é bem verdade que Dobzhansky toca neste ponto, como já vimos, mas o faz de modo superficial. Provine (1986) opina, por exemplo, que ele deve ter escrito o artigo antes do Congresso Internacional de Genética, isto é, antes de assistir à apresentação do trabalho de Sewall Wright, onde 
foi feita uma síntese dos mecanismos da evolução. A segunda dificuldade diz respeito ao fato de que Dobzhansky enfatiza a necessidade de se entender a genética das diferenças raciais e específicas para que se possa entender a dinâmica do processo de raciação e especiação justamente em organismos - besouros cuja genética era totalmente desconhecida e mais, organismos de difícil abordagem experimental. A oportunidade para resolver estas dificuldades viria justamente com a troca do material experimental que até então ele utilizara preferencialmente, isto é, a Drosophila melanogaster, por Drosophila pseudoobscura. Os trabalhos publicados com esta espécie, neste ano de 1933, referidos anteriormente, representaram o primeiro passo para a resolução dos problemas de genética da especiação e das diferenças raciais.

Em março de 1936, Dobzhansky e Alfred Sturtevant elaboraram um grande projeto para estudar populações naturais da mosca Drosophila pseudoobscura; o projeto tencionava estudar a evolução de populações naturais, particularmente dos processos evolutivos, através de experimentos de laboratório e da descrição da variabilidade genética presente nas populações. O projeto reunia, em síntese, a atividade do geneticista experimental e a do naturalista. Os estudos de evolução adquiriam um novo status, não mais exclusivamente descritivo, como na tradicional paleontologia. Para este projeto, eles contavam com o apoio de Sewall Wright, na solução dos problemas teóricos e no planejamento dos experimentos. Dobzhansky sabidamente tinha dificuldades com o tratamento quantitativo dos problemas de genética, enquanto Sturtevant era melhor versado nesta área. Mesmo assim, por iniciativa deste último, foi feito contato com Sewall Wright, em carta de 18 de março de 1936, onde ele dizia (Provine, 1986, p. 336): "Nós sentimos a necessidade da sua ajuda não somente na análise dos dados, mas também no planejamento de experimentos adequados."

É interessante registrar que, embora os frutos deste grande projeto tenham beneficiado decisivamente a Theodosius Dobzhansky, no que diz respeito à projeção internacional, foi na verdade Alfred Sturtevant quem deu os primeiros passos para que ele se concretizasse. Como resultado deste projeto, uma série de trabalhos conhecida como genética de populações naturais teve início em 1938 e se prolongou por mais de quarenta anos! Surpreendentemente, Sturtevant não figura como autor ou co-autor em nenhum deles.

$\mathrm{Na}$ década de 1930, desenvolvia-se um movimento, iniciado na década anterior, de unificação da biologia. O movimento, ainda que não tenha sido explicitado na maioria das vezes, tinha como suporte filosófico as idéias dos integrantes do Círculo de Viena, os quais defendiam a unificação nos métodos e na linguagem científica, rejeitando a metafísica. Este movimento na biologia ficou conhecido como síntese evolutiva, da qual resultou a chamada teoria sintética da evolução 
(uma excelente análise deste movimento pode ser encontrada em Mayr et al., 1998; Smocovitis, 1996). Dobzhansky é considerado um dos arquitetos da nova síntese; seu livro Genetics and the origin of species, cuja primeira edição é de 1937, teve papel fundamental na aceitação das novas idéias entre biólogos de diferentes áreas. O livro foi, sem dúvida, o mais influente publicado sobre biologia evolutiva nos primeiros cinqüenta anos do século XX. Nesta obra, Dobzhansky transformou as expressões matemáticas dos grandes teóricos da genética de populações (Wright, Fisher, Haldane) em uma linguagem acessível aos biólogos. Como salientaram Ayala et al. (1997), ele "vestiu as equações com história natural e genética de populações experimentais, estendendo a síntese à especiação e a outros problemas importantes omitidos pelos matemáticos".

Este livro, bem como a série de publicações intitulada Genetics of natural populations constituem a marca fundamental da contribuição de Dobzhansky para a síntese evolutiva e podem ser resumidos em dois pontos (Araújo, 1998): a investigação de populações naturais, portanto o estudo da evolução enquanto ela estava ocorrendo (uma área mais tarde denominada genética ecológica por E. B. Ford, na Inglaterra); e o uso do método experimental para testar teorias de evolução, com isto possibilitando a emergência do falsificacionismo popperiano nesta área. Dobzhansky deu-se conta disto, quando narrou suas reminiscências para a o Departamento de História Oral da Universidade de Columbia: "A genética é a primeira das ciências biológicas que alcançou uma posição na qual a física já está há muitos anos. Pode-se falar, justificadamente, em coisas tais como uma genética matemática, teórica, bem como uma genética experimental, tal como na física."

Theodosius Dobzhansky é considerado com justiça o agregador dos naturalistas e dos experimentalistas nas pesquisas sobre evolução. Podese afirmar que ele atraiu também os teóricos da evolução, ao aprender, com Sewall Wright, que um experimento de campo pode ser tão bem planejado como um experimento de laboratório. Sua influência foi enorme em vários países, inclusive no Brasil, onde esteve em várias oportunidades, as quais, se somadas, chegam a praticamente três anos. Aqui ele iniciou um grupo de pesquisadores da Universidade de São Paulo (USP) na área de genética evolutiva, com Drosophila; aqui ele ministrou conferências e cursos, influenciando toda uma geração de geneticistas. Aqui também ele se ligou afetivamente a vários personagens, com eles colaborou e com eles rompeu. Vários aspectos desta interação foram tratados por Moscoso (1992), Glick (1994), Araújo (1998), entre outros. 


\section{REFERÊNCIAS BIBLIOGRÁFICAS}

Allen, Garland E. 1994

Araújo, Aldo M. 1998

Ayala, Francisco J. e Fitch, Walter $M$. 1997

Bowler, Peter J. 1989

Behe, Michael 1997

Chandebois, Rosine 1993

Coleman, William 1977

Dobzhansky, T. 1937

Dobzhansky, T. 1933

Fisher, Ronald A. 1918

Glick, Thomas 1994

Huxley, Julian 1943

Kohler, Robert E. 1994

Krementsov, Nicolai L. 1994

Krug, Carlos A. 1932

Land, Barbara 1973

Martins, Lilian A. P. 1998

Mayr, Ernst e Provine, William B. (orgs.) 1998

Moscoso, Teodoro 1992
'Theodosius Dobzhansky, the Morgan Lab, and the breakdown of the naturalist/experimentalist dichotomy, 1927-1947'. Em M. B. Adams (org.), The evolution of Theodosius Dobzhansky. Princeton, Princeton University Press, pp. 87-98.

'A influência de Theodosius Dobzhansky no desenvolvimento da genética no Brasil'. Episteme, 3:7 pp. 43-54.

'Genetics and the origin of species: an introduction'.

Proceedings of the National Academy of Sciences, 94: pp. 7691-7.

Evolution: the bistory of an idea.

University of California Press.

A caixa-preta de Darwin: o desafio da bioquímica à teoria da evolução. Rio de Janeiro, Jorge Zahar Editor.

Para acabar com o darwinismo: uma nova lógica da vida. Lisboa, Instituto Piaget.

Biology in the nineteenth century: problems of form, function, and transformation. Cambridge, Cambridge University Press.

Genetics and the origin of species.

Fac-símile da 1aㅡ. ed., New York, Columbia University Press.

'Geographical variation in Lady-Beetles'.

The American Naturalist, 67, pp. 97-126.

'The correlation between relatives on the supposition of Mendelian inheritance', Transactions of the Royal Society of Edinburgh, 52:399-433.

'The Rockefeller Foundation and the emergence of genetics in Brazil, 1943-1960'. Em M. Cueto (org.), Missionaries of science-The Rockefeller Foundation in Latin America. Indiana, Indiana University Press, pp. 149-64.

Evolution: the modern synthesis.

New York. Harper \& Brothers Publishers.

'Fly room west: Dobzhansky, D. pseudoobscura and scientific practice'. Em M. B. Adams (org.), The Evolution of Theodosius Dobzhansky. Princeton, Princeton University Press, pp. 115-1287.

Dobzhansky and Russian entomology: the origin of his ideas on specie and speciation.' Em M. B. Adams (org.), The evolution of Theodosius Dobzhansky. Princeton. Princeton University Press, pp. 31-48.

'Sexto Congresso Internacional de Genética. Ithaca - Nova York, 24 a 30 de agosto de 1932'. Revista de Agricultura,7, pp. 446-54.

Evolution of a scientist: the two worlds of Theodosius Dobzhansky. Thomas Y. Crowell Co.

'Thomas Hunt Morgan e a teoria cromossômica: de crítico a defensor'. Episteme, 3:6, pp. 100-26.

The evolutionary synthesis - perspectives on the unification of biology. $2^{\mathbf{a}^{-}}$edição, com novo prefácio de, E. Mayr, Cambridge, Massachusetts. Harvard University Press.

Genetics and geneticists in Brazil: an essay about Latin American science and scientists. Tese de bacharelado. Harvard University.

Cambridge, Massachusetts. USA. 
ALDO MELLENDER DE ARAÚJO

Provine, William B. Sewall Wright and evolutionary biology.

1986 Chicago, The University of Chicago Press.

Provine, William B. The origins of theoretical population genetics.

1971 Chicago, The University of Chicago Press.

Smocovitis, Vassiliky B. Unifying Biology: the evolutionary synthesis and evolutionary Biology.

1996 Princeton, Princeton University Press.

Recebido para publicação em setembro de 2000.

Aprovado para publicação em junho de 2001. 